\title{
O Discurso da legitimação: a busca pelo espaço acadêmico da psicologia anomalística brasileira ${ }^{1}$
}

The speech of legitimation: the Brazilian anomalistic psychology and its search for academic space

\author{
Fernanda Loureiro Goulart ${ }^{2}$ \\ Léa Velho ${ }^{3}$
}

\section{RESUMO}

A psicologia anomalística é uma área nova na academia brasileira que busca estudar fenômenos anômalos a partir de um enfoque psicossocial, caracterizando-se como uma área que trava relações íntimas com a parapsicologia e com a psicologia em geral, especificamente com a psicologia social. No Brasil, um grupo têm-se destacado como propulsor dessa nova área, o Inter Psi - Laboratório de Psicologia Anomalística e Processos Psicossociais do Instituto de Psicologia da Universidade de São Paulo. Partindo do conceito de boundary work, este texto analisa artigos recentes publicados por membros do Inter Psi em periódicos de circulação entre psicólogos e parapsicólogos, identificando estratégias discursivas para legitimar os seus estudos nessas duas frentes. Em ambos os casos, conclui-se que o Inter Psi busca identificar lacunas na produção de conhecimento da psicologia e da parapsicologia, estabelecendo a psicologia anomalística como uma saída legítima para o preenchimento dessas lacunas. Conclui, ainda, que esse trabalho de demarcação de fronteiras se constitui como um espaço performativo de negociação dos limites da psicologia anomalística e da sua relação com a psicologia e com a parapsicologia.

Palavras-chave: Boundary work. Psicologia anomalística. Psicologia. Parapsicologia.

\begin{abstract}
Anomalistic psychology is a new are inside the Brazilian academic environment and it seeks to study anomalous phenomena through a psychosocial approach, being characterized as an area that establishes close contact with both parapsychology and psychology in general, more specifically social psychology. In Brazil, there is a group that has acted as a promoter of this new area, Inter Psi Laboratório de Psicologia Anomalística e Processos Psicossociais (Inter Psi - Laboratory of Anomalistic Psychology and Psychosocial Processes), from inside the Institute of Psychology of the Universidade de São Paulo. Using the concept of boundary work, this text analyses recent papers written by member of Inter Psi and published in journals that circulate among psychologists and parapsychologists, identifying discursive strategies to legitimate their studies in these two fronts. In both cases, we conclude that Inter Psi seeks to identify gaps in the knowledge production of psychology and parapsychology, establishing anomalistic psychology as a legitimate way to fill in these gaps. We conclude also that this boundary work can be seen as a performative way to negotiate the limits of anomalistic psychology and its connection to psychology and parapsychology.
\end{abstract}

Keywords: boundary work. Anomalistic psychology. Psychology. Parapsychology.

\footnotetext{
${ }^{1}$ Trabalho apresentado no dia 16 de outubro de 2013, no Grupo de Trabalho 5 - Inscrições e seus dispositivos: da produção científica à divulgação da ciência.

2 Doutoranda do Departamento de Política Científica e Tecnológica - UNICAMP. E-mail: fernandagoulart@ige.unicamp.br

3 Professora titular do Departamento de Política Científica e Tecnológica - UNICAMP. E-mail: velho@ige.unicamp.br
} 


\section{INTRODUÇÃO - PARAPSICOLOGIA E PSICOLOGIA ANOMALÍSTICA}

A parapsicologia é uma área que tem se encontrado em uma espécie de limbo de demarcação científica desde seu surgimento, durante fins do século XIX e início do século XX. Isso porque, mesmo tendo conseguido algumas conquistas em sua luta por legitimação (por exemplo, a Parapsychological Association, PA, maior associação de parapsicólogos no mundo, tornou-se parte da prestigiada American Association for the Advancement of Science, AAAS, na década de 1980, e foi aberta uma cátedra de parapsicologia na Universidade de Edimburgo em 1985), a parapsicologia não apresenta um alto grau de institucionalização acadêmica ao redor do mundo, e seu status de ciência ou não continua sendo ponto de discórdia entre cientistas e a opinião pública em geral. ${ }^{4}$

No Brasil, a história da parapsicologia é singular, devido aos embates entre parapsicólogos católicos e espíritas que marcou a área durante o século XX (HESS, 1991; MACHADO, 2005). A partir da década de 1990, houve uma busca por maior cientificidade entre grupos parapsicológicos brasileiros. Nesse contexto, surgiu o Inter Psi, um grupo de pesquisas em parapsicologia que buscou se inserir na academia.

O Inter Psi - Laboratório de Psicologia Anomalística e Processos Psicossociais é a encarnação atual de um grupo de pesquisas que nasceu como ECLIPSY - Instituto de Investigações Científicas em Parapsicologia, instituto particular e independente criado por Wellington Zangari em meados da década de 1990, em São Paulo, capital. Após entrar na Faculdade Anhembi-Morumbi como professor, em 1991, Wellington Zangari criou o InterPsi - Instituto de Pesquisas Interdisciplinares das Áreas Fronteiriças da Psicologia. O objetivo principal do grupo era estudar de forma interdisciplinar anomalias psicológicas, divididas em quatro

\footnotetext{
${ }^{4}$ A parapsicologia tem sido objeto de estudo da área de estudos sociais da ciência e da tecnologia, embora existam poucos casos. (COLLINS; PINCH, 1979) é um clássico da área, que discute os principais motivos de rejeição da cientificidade da parapsicologia por parte de cientistas hard. Allison (1979) e Gordon (1982) também discutem a rejeição à parapsicologia e seus motivos, enquanto Mauskopf e McVaugh (1980) fazem uma descrição mais longa e detalhada das tentativas de legitimação da parapsicologia americana durante a década de 1930. McClenon (1984) discute com mais detalhamento tanto a rejeição quanto as conquistas da parapsicologia, como a entrada da PA na AAAS. De qualquer forma, não se tem produzido sobre o assunto nas últimas décadas, muito porque a situação da legitimação da parapsicologia continuou estagnada: há parapsicólogos na academia, em geral tendo seus postos acadêmicos assegurados em áreas outras, e produzindo em parapsicologia como atividade secundária, com a parapsicologia tendo dificuldade em se estabelecer como uma área de pesquisas institucionalizada.
} 
grupos de pesquisa: experiências e fenômenos parapsicológicos, os chamados tratamentos alternativos, estados alterados de consciência, e simbolismo religioso e psicologia. Dos fins da década de 1990 até 2008, o Inter Psi esteve situado na PUCSP, com outro nome: Inter Psi - Grupo de Estudos de Semiótica, Interconectividade e Consciência. O grupo se realocou na PUC devido à entrada, em 1999, de Fátima Regina Machado - participante do Inter Psi desde seus tempos de ECLIPSY e esposa de Wellington Zangari - como pesquisadora e posteriormente professora da área de comunicação social. Na PUC, o Inter Psi esteve integrado ao Centro de Estudos Peirceanos, por sua vez parte do programa de pós-graduação em Comunicação e Semiótica.

A partir de seu ingresso na USP em 2008 como professor doutor do Departamento de Psicologia Social, no Instituto de Psicologia, Zangari propôs a abertura de um grupo de estudos de psicologia anomalística, que foi aceito e oficialmente aberto em 2010. Assim, o Inter Psi se relocou - pela, até aqui, última vez - para uma nova casa, sob o nome atual de Inter Psi - Laboratório de Psicologia Anomalística e Processos Psicossociais ${ }^{5}$.

Durante toda a sua história, o Inter Psi foi, portanto, um grupo diretamente envolvido na luta pela legitimação da parapsicologia. Como tal, estava inserido em uma controvérsia a respeito da cientificidade dessa área. Embora o Inter Psi continue ligado à parapsicologia (seus membros são membros da PA, por exemplo), o grupo atualmente se define como um grupo de psicologia anomalística ${ }^{6}$.

O que seria essa psicologia anomalística e qual sua relação com a parapsicologia? Segundo Fátima Machado (2010, p. 265):

A Parapsicologia, enquanto ciência, ocupa-se principalmente da realidade ontológica de psi, diferentemente do estudo aqui apresentado que se enquadra numa nova área da Psicologia, nomeada Psicologia Anomalística. A Psicologia Anomalística engloba os estudos da pesquisa psi e das outras experiências anômalas com enfoque privilegiado no sujeito dessas experiências, ou seja, nos aspectos psicológicos envolvidos nessas vivências e/ou resultantes delas.

\footnotetext{
${ }^{5}$ Ao longo de sua história, o grupo tem mantido números diferentes de membros, assim como perfis diferentes de participação. Hoje em dia, trata-se de um grupo formado exclusivamente por pesquisadores acadêmicos, que estão fazendo pesquisas na área de psicologia anomalística. No primeiro semestre de 2013, o grupo foi composto de dois doutores (Wellington Zangari e Fatima Machado), e outros dez membros regulares, todos mestrandos ou doutorandos (com exceção de uma aluna, recém-graduada, que prestaria exame de mestrado no fim de 2013).
} 
Psicologia anomalística seria, assim, o estudo sob o enfoque psicológico de experiências anômalas. Por sua vez, experiências anômalas são aquelas que se referem a fenômenos que não podem ser explicados por teorias usuais, ou experiências que destoam do consenso cultural sobre a realidade, como casos de telepatia, precognição, experiências fora do corpo, contatos com espíritos, contatos com alienígenas, entre outras (MACHADO, 2009, p. 463; MARTINS, 2011, p. 448). O termo psicologia anomalística passou a ser usado no Reino Unido a partir do livro de Leonard Zusne e Warren Jones, Anomalistic psychology: a study of extraordinary phenomena of behavior and experience, de 1982. Lá, tem conquistado espaço acadêmico cada vez maior, mas apresenta um viés mais crítico em relação à realidade ontológica de fenômenos psi (cf. HOLT et al., 2012). O objetivo principal é buscar compreender pelas teorias científicas em voga experiências ditas paranormais.

A utilização do termo psicologia anomalística pelo Inter Psi pode sofrer resistência de parapsicólogos tradicionais (ZANGARI; MACHADO, 2012, p. 66), assim como demanda explicação de sua existência para psicólogos. Na busca pela legitimação dessa nova área no Brasil, os membros do Inter Psi fazem uso de uma série de estratégias, como a defesa em específico do nome, a criação das bases epistemológicas da área, a produção em si do conhecimento, a tentativa de angariar recursos para pesquisas e a busca por manter contatos com diferentes grupos acadêmicos (como psicólogos anomalísticos do Reino Unido), formando uma rede específica de pesquisa em psicologia anomalística. Uma dessas estratégias é a identificação da área de concentração das pesquisas e a defesa da importância científica dos estudos dos tópicos escolhidos.

Como um grupo tradicionalmente ligado à parapsicologia, mas recentemente definido como um grupo de psicologia, o Inter Psi precisa defender os limites de sua área para audiências tanto de psicólogos quanto de parapsicólogos. O presente texto busca analisar como os membros do Inter Psi se utilizam de recursos discursivos para defender a importância dos seus estudos frente a essas duas audiências, respondendo de certa forma à pergunta "por que deve existir uma área chamada psicologia anomalística?" tanto para psicólogos quanto para parapsicólogos. 
Para tanto, escolhemos publicações em periódicos de circulação entre esses grupos, todos produzidos a partir de 2008, ano em que Zangari se tornou professor da USP e submeteu a proposta de abertura do Inter Psi na instituição, ou seja, a partir do momento em que o Inter Psi se tornou de fato um grupo de psicologia anomalística. Como Zangari também publica e orienta pesquisas mais voltadas para a área de psicologia da religião, foram deixados de lado artigos especificamente sobre espiritualidade e crenças religiosas produzidos por membros do Inter Psi.

\section{UM CASO DE BOUNDARY WORK}

Thomas Gieryn $(1983,1999)$ se ocupou detidamente do estudo de casos de controvérsias sobre demarcação científica. Ele utiliza um conceito bastante útil na discussão da demarcação científica, que é o de boundary work (que podemos traduzir como um "trabalho de demarcação de fronteiras"). Gieryn o define como uma atividade rotineira dos cientistas de demarcação do que é ciência e, por tabela, do que não é ciência. Mais especificamente, é "(...) the discursive attribution of selected qualities to scientists, scientific methods, and scientific claims for the purpose of drawing a rhetorical boundary between science and some less authoritative residual non-science"'" (GIERYN, 1999, pp. 4-5).

Gieryn estuda casos em que cientistas defendem uma visão específica de ciência ou de suas áreas em específico, geralmente estabelecendo fronteiras em diferentes frentes. Essa fabricação de fronteiras múltiplas evidencia características contraditórias da ciência em geral. Um caso representativo é o de John Tyndall (GIERYN, 1999, pp. 37-64), que buscou definir a ciência em contraposição à religião e à engenharia, dotando-a de características diferentes em cada caso (empírica e prática em relação à religião, metafísica e poética em relação à engenharia).

Conquanto Gieryn se ocupe de casos de controvérsias científicas, Felicity Mellon (2004) afirma que o conceito de boundary work não precisa ser aplicado somente a esses casos. Ela estudou, por exemplo, casos de demarcação de fronteiras em livros-texto de física, argumentando que situações não controversas 
evidenciam suposições e expectativas culturais mais gerais a respeito do que é ciência (MELLOR, 2003, p. 520).

Os textos de psicologia anomalística se tratam de um meio termo entre os casos analisados por Gieryn e Mellon. Não estamos frente a uma controvérsia científica per se, mas os textos também não se tratam de conhecimento já incorporado aos cânones científicos, como no caso dos livros-texto. São, por exemplo, textos para pesquisadores, não para o público em geral. Trata-se de um caso particular de uma área que nasceu ligada a uma outra área controversa, a parapsicologia, mas busca um nicho acadêmico inserida dentro de uma área mais institucionalizada, que é a psicologia, especificamente a psicologia social. Não deixa de ser uma espécie de divulgação científica, mas para estabelecer as fronteiras da área em relação à psicologia e à parapsicologia.

No caso do Inter Psi, pode-se dizer que há um trabalho de demarcação de fronteiras sutil, que tem por objetivo apontar lacunas na produção do conhecimento tanto da psicologia quanto da parapsicologia, lacunas estas que podem ser preenchidas pelas pesquisas em psicologia anomalística.

\section{FRENTE AOS PSICÓLOGOS}

A partir de 2008, foram encontrados cinco artigos (MACHADO, 2010; MARALDI; ZANGARI, 2012; MARALDI; ZANGARI; MACHADO, 2011; MARTINS, 2011; MARTINS; ZANGARI, 2012) publicados em periódicos de circulação em psicologia, ambos indexados. Quatro artigos foram publicados no Boletim da Academia Paulista de Psicologia, com classificação Qualis B2 para psicologia, e um artigo saiu na Revista de Psiquiatria Clínica, publicada pela Faculdade de Medicina da Universidade de São Paulo, também classificada B2 para psicologia.

Por meio da leitura dos artigos, constata-se que o discurso da importância da área frente a psicólogos se foca em três elementos. Em primeiro lugar, os textos afirmam que é necessário estudar experiências anômalas devido à sua alta incidência na população, tanto no Brasil quanto em outros países. Machado (2010,

p. 463), Maraldi, Zangari e Machado (2011, p. 394), Martins (2011, pp. 448-9) e Martins e Zangari (2012, p. 198) se utilizam desse recurso. A tese de doutoramento 
de Fátima Regina Machado (2009), por exemplo, apresenta a pesquisa feita pela autora com 306 respondentes, estudantes universitários, dos quais 82,7\% disse ter tido pelo menos uma experiência anômala na vida. Os resultados da tese foram apresentados também em Machado (2010). Dos cinco artigos aqui analisados, Martins e Zangari (2012), Maraldi, Zangari e Machado (2011) e Maraldi e Zangari (2012) fizeram referência à tese de Machado.

Em segundo lugar, os textos apontam a importância de estudar experiências anômalas para melhor compreender o funcionamento psicológico humano, observando a incompletude das teorias científicas vigentes (ou seja, apontam lacunas na produção de conhecimento da psicologia). Machado (2010, p. 463), Martins (2011, pp. 448-9) e Martins e Zangari (2012, p. 198) chamam a atenção explicitamente para a corrente limitação da psicologia ao não levar em conta as experiências anômalas, enquanto Maraldi, Zangari e Machado (2011) afirmam que "o estudo das funções psicológicas e sociais das crenças e experiências relacionadas a fenômenos supostamente paranormais representa, em si mesmo, um esforço legítimo por parte de qualquer estudioso da Psicologia ou das demais ciências sociais" (MARALDI; ZANGARI; MACHADO, 2011, p. 395) ressaltando sua importância para o conhecimento mais pleno da condição humana.

Em terceiro lugar, os textos apontam a demanda clínica por conhecimento a respeito de experiências anômalas (observando uma lacuna na formação profissional, já que os psicólogos clínicos em geral não estão preparados para lidar com as experiências anômalas dos pacientes pela falta de discussões sobre o tópico nas universidades). Neste contexto, a discussão mais importante é a relação entre experiências anômalas e psicopatologias, acentuando-se a necessidade de se estudar os tópicos da psicologia anomalística para ajudar no caso de diagnósticos diferenciais. Machado (2010, p. 464), Martins (2011, pp. 449), Martins e Zangari (2012, p. 198) e Maraldi e Zangari (2012, p. 449) fazem referência à importância dos estudos sobre experiências anômalas para a prática clínica.

Martins e Zangari (2012, p. 198) oferecem uma síntese dos pontos presentes nos textos:

Quanto à sua relevância científica, as experiências anômalas são pessoal e culturalmente impactantes, além de altamente prevalentes. Em um estudo com voluntários brasileiros, por exemplo, encontrou-se que $82,7 \%$ dos 306 respondentes afirmaram ter vivenciado ao menos uma experiência anômala das investigadas na pesquisa (experiências de tipo extrassensório-motoras: 
experiências telepáticas, precognitivas e psicocinéticas). Por sua vez, as teorias científicas permanecem bastante incompletas ao desconsiderar tais experiências, dado que podem sinalizar lacunas de conhecimento sobre o funcionamento psicológico humano (e.g., sobre alucinações em populações não clínicas). Finalmente, desenha-se uma relevância clínica, pois muitos protagonistas recebem diagnósticos equivocados de profissionais de saúde despreparados para lidar com as experiências. Adicionalmente, alguns tipos de experiências anômalas podem promover bem-estar psicológico de modo especial, como uma radical, acelerada, positiva e estável reestruturação da vida do protagonista. Desse modo, experiências anômalas e temas associados têm sido objeto de crescente interesse científico, inclusive no Brasil, com pesquisas em diversos domínios, incluindo diagnóstico diferencial e adaptação de instrumentos relacionados.

\section{FRENTE AOS PARAPSICÓLOGOS}

Foram encontrados três artigos publicados em periódicos de circulação entre meios parapsicológicos (MARALDI; MACHADO; ZANGARI, 2010; MARTINS, 2013; ZANGARI; MACHADO, 2012), não indexados. Os artigos foram publicados respectivamente no Journal of Scientific Exploration (da Society for Scientific Exploration), no Paranthropology e no Journal of Parapsychology (publicado pela $\mathrm{PA}$ ). Embora apenas o último se trate de um periódico de parapsicologia especificamente, os outros dois são destinados à publicação de estudos sobre fenômenos e experiências paranormais. Ou seja, os três são de circulação entre grupos que se ocupam do estudo do paranormal.

Os artigos de Maraldi, Machado e Zangari (2010) e Martins (2013) se caracterizam pela proximidade aos textos dos autores que foram publicados em periódicos de circulação entre psicólogos. O que pode ser observado e que faz referência aos estudos prévios em parapsicologia é a referência à importância de se estudar o paranormal a partir da psicologia - mais especificamente a partir da psicologia social - para que se compreenda melhor os fenômenos em geral (observando uma lacuna na produção de conhecimento da parapsicologia). Maraldi, Machado e Zangari (2010, p. 184) comentam a respeito do estudo dos pesquisadores da chamada psychical research (origem da parapsicologia) a respeito da mediunidade: "One evident problem (...) was the emphasis on individual aspects of mediumship, that is on the study of intrapsychic and unconscious processes in mediums. This approach neglected the power of culture and society in modeling 
beliefs and experiences associated with mediumistic phenomena", afirmando depois que a ótica exclusivamente psicopatológica (lacuna da psicologia) e a exclusivamente intrapsíquica (lacuna da parapsicologia) ainda predominam nos estudos sobre o tema, e é necessário abrir caminho para uma ótima psicossocial (MARALDI; MACHADO; ZANGARI, 2010, pp. 193-4).

No mesmo sentido, Martins (2013, pp. 16-7) identifica duas dimensões no estudo científico de experiências anômalas: a fenomenológica e a ontológica, salientando que são dois modelos epistemológicos distintos e não é possível tomar apenas um deles como válido. Não é viável, segundo ele, adotar um enfoque puramente intrapsíquico para as experiências, ignorando uma abordagem psicossocial. Assim, os textos atuam contra a resistência à psicologização do estudo do paranormal, advogando em favor do valor de um enfoque psicossocial.

O texto de Zangari e Machado (2012), entretanto, dialoga mais proximamente com os parapsicólogos, já que se trata de uma avaliação dos autores a respeito do campo da parapsicologia no Brasil em uma edição especial do Journal of Parapsychology, comemorando o septuagésimo quinto aniversário do periódico (contendo textos curtos de parapsicólogos ao redor do mundo respondendo à questão: onde estará a parapsicologia daqui a 25 anos?). No texto, os autores afirmam crer que a parapsicologia brasileira irá desaparecer em 25 anos. Entretanto, caracterizam esse desaparecimento como paradoxal, pois significaria exatamente 0 renascimento da pesquisa psi na academia brasileira (ZANGARI; MACHADO, 2012, p. 67). Para os autores, é impossível eliminar a conotação negativa do termo "parapsicologia" no Brasil devido ao histórico da área no país e ao uso contínuo do termo por pessoas que oferecem trabalho clínico sem terem qualquer formação universitária em saúde mental. A saída foi adotar um outro termo:

Thus, the solution found by many of those who are interested in the scientific study of psi was to move away from everything that is commonly associated with parapsychology in Brazil. And some researchers realized that, to study paranormal claims and correlated subjects, they should assume a frankly skeptical attitude. For this reason, the term "anomalistic psychology" was recently introduced and has been propulgated in Brazil, especially in the academy, to denote an area of study of paranormal claims from a skeptical perspective. However, researchers in anomalistic psychology are not closed to the study of the psi hypothesis, although they emphasize the psychological processes underlying paranormal claims. (...) Thus, at least in Brazil, the field of anomalistic psychology has represented not only the opening for the academic study of psychological experiences, beliefs, and/or paranormal claims, but also the normalization of the scientific study of the psi hypothesis. 
Assim, os autores apresentam a existência da psicologia anomalística brasileira como uma resposta ao fechamento da academia em relação à parapsicologia. A psicologia anomalística se apresenta como uma normalização do estudo do paranormal.

\section{CONCLUSÕES}

Em primeiro lugar, cumpre notar que o Inter Psi tem publicado mais em revistas com audiência de psicólogos do que de parapsicólogos. Isso é também um reflexo da busca por institucionalização, já que os membros são levados a publicar em revistas indexadas, o que não é o caso do Journal of Parapsychology e do Journal of Scientific Exploration. A permanência de publicação na área de parapsicologia, contudo, reflete a vontade do grupo em manter vias de diálogo em diferentes frentes.

Para manter essas diferentes frentes, o grupo mostra análise dos campos: identifica lacunas e busca colocar a psicologia anomalística como a provedora de respostas para essas questões. Assim, demonstra um trabalho de fronteiras ao estilo dos casos estudados por Gieryn (1982, 1999). Se o discurso do Inter Psi se modifica frente a audiências de psicólogos e parapsicólogos, isso se deve não apenas ao fato de que a ciência apresenta fronteiras geralmente fluidas, mas também a característica peculiar da psicologia anomalística que é encontrar-se em um ambiente cinzento entre a psicologia e a parapsicologia, buscando manter diálogos com as duas áreas.

Pode-se argumentar, ainda, que o trabalho de fronteiras aqui estudado se refere a uma entre várias estratégias de ordenação, conforme Law (2004) entende o trabalho de ordenação de mundo. Temos um trabalho retórico que é performativo (Callon, 2006), no sentido em que ajuda a criar uma imagem da psicologia anomalística, da parapsicologia e da psicologia que ajudam a informar os profissionais da área e, por consequência, a imaginação pública a respeito dessas áreas. 
O Inter Psi apresenta a psicologia anomalística como uma área especialmente promissora porque pode contribuir para solucionar lacunas no conhecimento psicológico e parapsicológico, tornando-se ocupante de um nicho acadêmico específico.

No entanto, as diferenças entre os discursos - notadamente a forte correlação entre psicologia anomalística e parapsicologia presente em Zangari e Machado (2012), mas não presente nos textos de periódicos da área de psicologia, não se caracteriza apenas como marca do caráter múltiplo da ciência e da dificuldade em demarcar fronteiras. Conforme Mellon (2004, p. 521) sugere, o trabalho de demarcação de fronteiras se caracteriza também como um espaço de negociação de contradições. Em um esforço performativo, a demarcação rotineira de fronteiras contribui para que os pesquisadores organizem o mundo científico do qual falam, negociem suas bases epistemológicas e avaliem cotidianamente a produção das suas áreas de pesquisa.

No caso do Inter Psi, o grupo define uma psicologia anomalística que significa a entrada da parapsicologia na academia brasileira, mas uma entrada feita sob condições específicas, que são o foco em processos psicológicos e uma postura cética (o que tem sido feito, tomando por base os textos aqui analisados, que se concentram no estudo psicossocial de experiências anômalas). Ao preencher esses pré-requisitos, o grupo mantém a abertura para o estudo ontológico de fenômenos paranormais (a hipótese psi de que falam Zangari e Machado, 2012, p. 16).

Em todo o caso, é importante notar que estamos tratando de textos de publicação recente. Será interessante observar as publicações posteriores de membros do Inter Psi, buscando avaliar se eles publicarão também pesquisas a respeito da ontologia de fenômenos paranormais, e se essas pesquisas encontrarão abertura em periódicos de circulação entre psicólogos. 


\section{REFERÊNCIAS}

ALLISON, Paul D. Experimental parapsychology as a rejected science" In: WALLIS, Roy (ed.). On the margins of science: the social construction of rejected knowledge. Sociological Review monograph. Keele: University of Keele Press, 1979, pp. 271-292.

CALLON, Michel. What does it mean to say that economics is performative? In: MacKENZIE, D.; MUNIESA, F.; e SIU, L; Do economists make markets? Princeton: Princeton University Press, 2006, pp. 311-357.

COLLINS, H. M.; PINCH, T. J. The construction of the paranormal: nothing unscientific is happening. In: WALLIS, Roy (ed.). On the margins of science: the social construction of rejected knowledge. Sociological Review monograph. Keele: University of Keele Press, 1979, pp. 237-270.

GIERYN, Thomas F. Boundary-work and the demarcation of science from nonscience: strains and interests in professional ideologies of scientists". American Sociological Review, vol. 48, 1983, pp. 781-195.

GIERYN, Thomas F. Cultural boundaries of science: credibility on the line. Chicago/London: The University of Chicago Press, 1999.

GORDON, Michael D. How socially distinctive is cognitive deviance in an emergent science? The case of parapsychology. Social Studies of Science, vol. 12, 1982, p. 151-165.

HESS, David. Spirits and scientists: ideology, spiritism and Brazilian culture. University Park: The Pennsylvania State University, 1991.

HOLT, Nicola J., SIMMONDS-MORE, Christine, LUKE, David and FRENCH, Christopher C. Anomalist psychology. Palgrave Insights in Psychology. Palgrave, MacMillan, 2012, 252 pp.

LAW, John. Organizing modernity. Oxford/Cambridge: Blackwell, 1994.

MACHADO, Fátima R. Parapsicologia no Brasil: entre a cruz e a mesa branca. Boletim Virtual de Pesquisa Psi, site www.pesquisapsi.com, v. 2, n.2, 2005.

MACHADO, Fátima R. Experiências anômalas na vida cotidiana: experiências extrassensório-motoras e sua associação com crença, atitudes e bem-estar subjetivo. Tese de doutorado. São Paulo: Instituto de Psicologia da Universidade de São Paulo; 2009.

MACHADO, Fátima R. Experiências anômalas (extra-sensório-motoras) na vida cotidiana e sua associação com crenças, atitudes e bem-estar subjetivo. Boletim da Academia Paulista de Psicologia, v. 30, 2010, p. 462-483. 
MARALDI, Everton O.; MACHADO, Fátima R.; ZANGARI, Wellington. Importance of psychosocial approach for a comprehensive understanding of mediumship. Journal of Scientific Exploration, v. 24, 2010, p. 181-196.

MARALDI, Everton O.; ZANGARI, Wellington. Funções projetivas e terapêuticas das práticas dissociativas em contexto religioso. Boletim da Academia Paulista de Psicologia, v. 32, 2012, p. 424-452.

MARALDI, Everton O.; ZANGARI, Wellington; MACHADO, Fátima R. A psicologia das crenças paranormais: uma revisão crítica. Boletim da Academia Paulista de Psicologia, v. 31, 2011, p. 394-421.

MARTINS, Leonardo B. Ainda um mito moderno? A compreensão junguiana de experiências anômalas contemporâneas revisitada. Boletim da Academia Paulista de Psicologia, v. 31, 2011, p. 447-464.

MARTINS, Leonardo B. Epistemological, methodological and ethical aspects of conducting interviews about anomalous experiences. Paranthropology, v. 4, 2013, p. 15-24.

MARTINS, Leonardo B; ZANGARI, Wellington. Relações entre experiências anômalas tipicamente contemporâneas, transtornos mentais e experiências espirituais. Revista de Psiquiatria Clínica (impresso), v. 39, 2012, p. 198-202.

MAUSKOPF, Seymour H.; McVAUGH, Michael R. The elusive science: origins of experimental psychical research. Baltimore: The Johns Hopkins University Press, 1980.

MELLOR, Felicity. Between fact and fiction: demarcating science from non-science in popular physics books. Social Studies of Science, Vol. 33, No. 4, Agosto de 2003, pp. 509-538.

McCLENON, James. Deviant science: the case of parapsychology. Filadélfia: University of Pennsylvania Press, 1984.

ZANGARI, Wellington; MACHADO, Fátima R. The paradoxal disappearance of parapsychology in Brazil. Journal of Parapsychology, v. 76, 2012, p. 66-67. 